\title{
Modulation Behaviors, Conductivities, and Carrier Dynamics of Single and Multilayer Graphenes
}

\author{
Emine Kaya ${ }^{1}$, Nurbek Kakenov², Coskun Kocabas², Hakan Altan ${ }^{3}$, Okan Esenturk ${ }^{1, *}$ \\ ${ }^{1}$ Department of Chemistry, Middle East Technical University, 06800 Ankara, Turkey \\ ${ }^{2}$ Department of Physics, Bilkent University, 06800 Ankara, Turkey \\ ${ }^{3}$ Department of Physics, Middle East Technical University, 06800 Ankara, Turkey \\ *eokan@metu.edu.tr
}

\begin{abstract}
Time domain and time resolved terahertz studies of single- and multi-layer graphene (SLG and MLG) samples and modulator devices will be presented. A high performance up to $100 \%$ of modulators were observed with the devices even at very low voltages. High modulation depth over such a broad spectrum and simple device structure brings significant importance toward application of this type of device in $\mathrm{THz}$ and related technologies. In addition, conductivities of SLG and MLG devices were also investigated and a change in behavior was observed as the layer thickness increased. The charge carriers dynamics of the samples with pulp fluence and color was also highly interesting.
\end{abstract}

\section{INTRODUCTION}

$\mathrm{S}_{\mathrm{i}}^{\mathrm{s}}$ NCE its discovery in 2004, graphene are of great interest in many fundamental science areas due to its exotic properties such as massless particles, RT quantum Hall effect, and micrometer-long mean free path.[1] Its gapless nature results almost constant absorption in the electromagnetic spectrum and makes it more sensitive to Terahertz (THz) region of the spectrum rather than IR and optical range.[2] It is becoming a promising material for $\mathrm{THz}$ devices as active filters, switches, and modulators in the future technologies.

Research in $\mathrm{THz}$ science and technology has exploded with increased accessibility to $\mathrm{THz}$ region. In turn this led to improvements in $\mathrm{THz}$ instrumentation and has created new application areas such as astronomy, biomedicine, defense, medical imaging, drug detection, communications, nondestructive testing and noncontact conductivity measurements. Since $\mathrm{THz}$ light can penetrate through most materials opaque to UV and visible, it provides excellent spectroscopic data of samples even under concealed environments. With terahertz's low energy, thus, long wavelength photons, it is possible to characterize both static and dynamic properties of diverse range of materials. Many emerging technologies require an ability to control and manipulate intensity and phase of $\mathrm{THz}$ waves. These technologies would benefit greatly from a $\mathrm{THz}$ modulators that have a simple device structure with efficient modulation. Applications of these devices include: power stabilization of $\mathrm{THz}$ sources in spectroscopy, carrier-wave modulation for $\mathrm{THz}$ band communication systems, and switches for coded aperture $\mathrm{THz}$ imaging. However, $\mathrm{THz}$ wave modulators studied to date generally exhibit compromised performance between modulation depth, broadband operation, signal attenuation, polarization dependence, on-chip integrability and structural complexity.

The devices designed in this study consists of SLG or MLG on top of quartz or a thin polymer substrate with gold electrodes for electrical contact. Graphene layer also includes ionic dopants. This design eliminates the strong spectral dependence caused by substrate effects. Polymer substrate also offers the opportunity of flexible devices.
This study will present characterization of SLG and MLG based broadband THz modulator devices and characterization of conductivities and carrier dynamics of graphene samples, in which the number of graphene layers, the type of underlying substrate, pump color, and fluence were varied to determine the frequency dependent conductivity behaviors and dominant mechanisms responsible for the carrier relaxation and cooling dynamics.
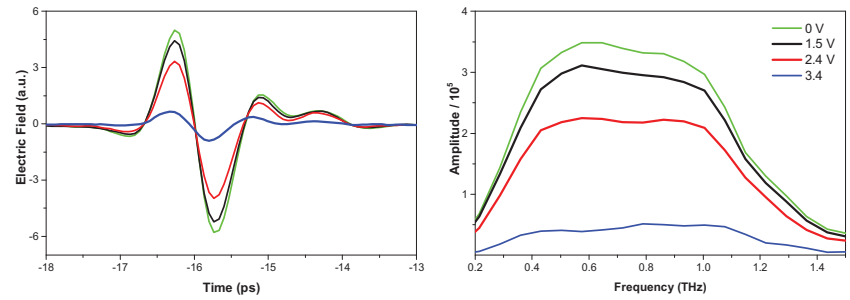

Fig. 1. Voltage dependent time domain profile and amplitude change of MLG sample with ca 60 layers in $0.2-1.5 \mathrm{THz}$ frequency range for representative voltages as an example.

\section{RESULTS}

Figure 1 represents an example of voltage dependent amplitude modulation behavior of a MLG device with ca. 60 layers. Behavior highly depends on the layer numbers of the devices and their conductivities. The SLG device provided ca. $50 \%$ amplitude modulation. On the other hand, the MLG devices provided a tunable amplitude modulation up to almost $100 \%$ at operation voltages below $3.5 \mathrm{~V}$. The effect of increased layer thickness on the modulation and other aspects of the device will also be presented along with the voltage dependence. Here, the modulation is frequency independent and bandwidth appears to be limited only by the instrument.

Figure 2 presents frequency dependent conductivity of the samples. As layer number increases conductivities of the samples increase and the frequency dependences change its behavior from Drude-Smith to Drude. Substrate also affects the behavior of the SLG samples. The relaxation dynamics of MLG samples revealed that a hotter distribution of carriers can be achieved either by increasing the absorbed photon density or photon energy. Further details of conductivities and carrier dynamics will also be presented.
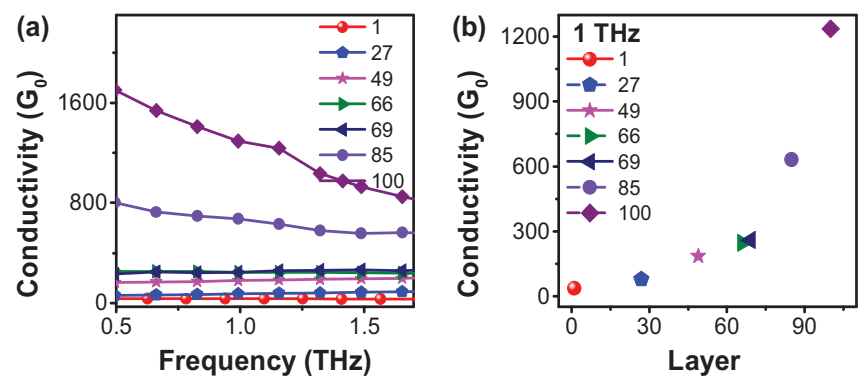

Fig. 2. (a) Frequency dependent conductivity behavior of SLG and MLG samples, (b) Conductivities at $1 \mathrm{THz}$. 
As a model 2D material, it is important to understand its nonequilibrium behavior for high speed electronic and photonic applications. We have investigated the photoconductivty of single- and multi-layer graphenes by optical pump terahertz probe measurements with time resolved Terahertz spectrometer. The results reveal the relaxation dynamics of the carrier upon photo excitation and its relation to the layer thickness.

Figure 3 presents the time resolved terahertz spectra of a) single layer and b) multi-layer graphene sample before and after excitation. Main difference between the single and multilayer graphene samples is their response to photoexcitation. The $\mathrm{THz}$ transmission increased in single layer graphene while it was decreased in multilayers.

The observed increase in the $\mathrm{THz}$ transmission of the single layer sample is due to a decrease in conductivity upon photoexcitation. This observed behavior is similar to a metal. Here, increased scattering process become a dominant component compared to the increase in carrier number. This result not only suggest a high initial doping level of single layer graphene. Calculations showed a Fermi energy, $\mathrm{E}_{\mathrm{F}}$ of $610 \mathrm{meV}$ with corresponding carrier density of $2.7 \times 10^{13}$.

The optical photoexcitation of MLG samples resulted decrease in their $\mathrm{THz}$ transmissions. There was no observable change in shape of the profile and no shift in time domain with optical pumping. This is an indication of dominant dispersionless real conductivity with may be a negligible imaginary part. After the initial carrier generation, the $\mathrm{THz}$ amplitude recovered fairly quickly with initial decay times less than 20 ps. Initial change in $\mathrm{THz}$ transmission upon photoexcitation depended almost linearly on the thickness or layer number of the MLG samples while the initial fast decay times increased as an almost exponential behavior. In all layers, a significant amount of long lived carriers left beyond $100 \mathrm{ps}$ due to a slow second decay rate. The amount of long lived carriers appears to be proportional to the initial maximum change in $\mathrm{THz}$ transmission, or carrier number. a)

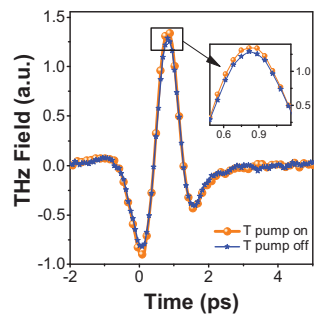

b)

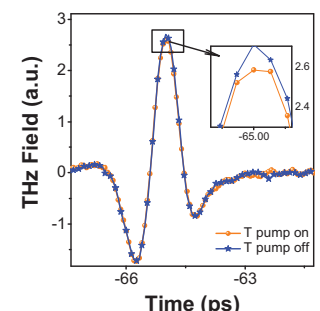

Fig. 3. THz time domain profiles of (a) single layer and b) 27 multi-layer graphenes before (blue stars) and $3.5 \mathrm{ps}$ after (orange circles) photoexcitation.

\section{SUMMARY}

In this study behavior of single and multi-layer graphene modulator with voltage change were presented. Tunable modulation behaviors up to ca. $100 \%$ were observed for multilayer graphenes while ca. 50\% modulation was achieved with single layer one. In addition, a parabolic increase in conductivities was observed with increase in layer numbers. Photoconductivity studies revealed two distinct behaviors. The
$\mathrm{THz}$ transmission of single layer sample was increased with optical pump, suggesting a high original doping level and metallic character. On the other hand, the terahertz transmission decreased with the optical pumping of the multilayer samples, suggesting a semiconductor type behavior. After an initial fast decay with a decay rate less than $20 \mathrm{ps}$, the recombination rate slowed down and a significant amount of long lived carriers were observed even beyond 100ps. The amount of long lived carriers appears to be proportional with initial change, hence, the layer number.

\section{REFERENCES}

[1]. Novoselov, K. S., Geim, A. K., Morozov, S. V., Jiang, D., Zhang, Y., Dubonos, S. V., Grigorieva, I. V., \& Firsov, A. A. Electric field effect in atomically thin carbon films. Science. 306, 666-669, 2004.

[2]. Dawlaty, J. M., Shivaraman, S., Chandrashekhar, M., Rana, F., \& Spencer, M. G. Measurement of ultrafast carrier dynamics in epitaxial graphene. Appl. Phys. Lett. 92, 42116, 2008. 